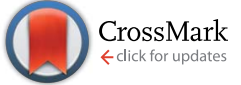

Cite this: J. Mater. Chem. A, 2015, 3, 7368

Received 1st February 2015

Accepted 15th February 2015

DOI: $10.1039 / \mathrm{cta}$ ta00829h

www.rsc.org/MaterialsA

\section{All conducting polymer electrodes for asymmetric solid-state supercapacitors $\uparrow$}

\begin{abstract}
Narendra Kurra, Ruiqi Wang and H. N. Alshareef*
In this study, we report the fabrication of solid-state asymmetric supercapacitors (ASCs) based on conducting polymer electrodes on a plastic substrate. Nanostructured conducting polymers of poly(3,4ethylenedioxythiophene), PEDOT, and polyaniline (PANI) are deposited electrochemically over Au-coated polyethylene naphthalate (PEN) plastic substrates. Due to the electron donating nature of the oxygen groups in the PEDOT, reduction potentials are higher, allowing it to be used as a negative electrode material. In addition, the high stability of PEDOT in its oxidised state makes it capable to exhibit electrochemical activity in a wide potential window. This can qualify PEDOT to be used as a negative electrode in fabricating asymmetric solid state supercapacitors with PANI as a positive electrode while employing polyvinyl alcohol (PVA)/ $\mathrm{H}_{2} \mathrm{SO}_{4}$ gel electrolyte. The ASCs exhibit a maximum power density of $2.8 \mathrm{~W} \mathrm{~cm}^{-3}$ at an energy density of $9 \mathrm{~mW} \mathrm{~h} \mathrm{~cm}^{-3}$, which is superior to the carbonaceous and metal oxide based ASC solid state devices. Furthermore, the tandem configuration of asymmetric supercapacitors is shown to be capable of powering a red light emitting diode for about 1 minute after charging for 10 seconds.
\end{abstract}

\section{Introduction}

Flexible electronic devices have increased demand for the development of light-weight and solid state energy storage units on flexible platforms such as plastic substrates. ${ }^{1-3}$ Supercapacitors, also called electrochemical capacitors, have become potential energy storage units due to their higher energy density compared to conventional electrostatic capacitors and higher power density compared to batteries along with the fast chargedischarge rates and long cycle life. ${ }^{3-7}$

Carbonaceous materials with a tunable surface area in different morphologies have been employed as electrodes for electrochemical double layer capacitors (EDLCs) ${ }^{8-11}$ Since the charge is stored through the formation of electrochemical double layers, capacitance values are typically lower for these carbonaceous materials compared to the pseudocapacitors that involve Faradaic reactions for storing the charge., ${ }^{2,8,12}$ In this context, several pseudo-capacitive materials such as transition metal oxides/hydroxides ${ }^{12,13}$ and conducting polymers ${ }^{2,3,14}$ have been employed to enhance the capacitance values due to their fast surface redox reactions. Besides the capacitance, the energy density $\left(E=1 / 2 C V^{2}\right)$ of a supercapacitor is determined by the square of the operating potential window of the electrolyte. ${ }^{6}$ Hence, non-aqueous electrolytes (organic and ionic liquids) are

Materials Science and Engineering, King Abdullah University of Science and Technology (KAUST), Thuwal 23955-6900, Saudi Arabia.E-mail: husam.alshareef@ kaust.edu.sa

$\dagger$ Electronic supplementary information (ESI) available: Fig. S1-S6 and Table S1. See DOI: 10.1039/c5ta00829h preferred due to their wide electrochemical potential windows (3-4 V) over the aqueous electrolytes $(1 \mathrm{~V})$ in obtaining superior energy density values. However, non-aqueous electrolytes suffer from poor ionic conductivity, flammability and high cost. ${ }^{15}$ Thus, there is an urge to extend the electrochemical potential window of aqueous electrolytes beyond $1 \mathrm{~V}$ by embracing the asymmetric design of electrode materials. ${ }^{15-18}$

Mostly, carbonaceous materials have been employed as negative electrode materials, while metal oxides/hydroxides and conducting polymers have been used as positive electrode materials in designing asymmetric supercapacitors (ASCs) employing liquid and solid electrolytes. ${ }^{15-20}$ Despite the higher potential window of asymmetric supercapacitors, the cell capacitance, $C_{\mathrm{T}}$, is limited by the lower capacitance electrode, mostly the capacitance of the negative carbonaceous electrode material, $C_{-},\left(1 / C_{\mathrm{T}}=1 / C_{+}+1 / C_{-}\right) .{ }^{16-21}$ In order to boost the cell capacitance of the ASC, metal oxides such as $\mathrm{Fe}_{2} \mathrm{O}_{3}$ and $\mathrm{V}_{2} \mathrm{O}_{5}$ have been employed as negative electrode materials that exhibit reversible redox reactions. ${ }^{22-25}$ However, the poor conducting nature of these oxides would result in the lower values of power density, and they should be synthesized in the form of composites with carbonaceous materials for better performance. ${ }^{15}$

Conducting polymers (CPs) have become attractive candidate materials for supercapacitors due to their many salient characteristics such as high electrical conductivity, ease of solution processing, low cost, electrochemical stability and reversibility between redox states through doping/dedoping processes. ${ }^{\mathbf{2 6 - 2 9}}$ Conducting polymer supercapacitors have been 
broadly classified into three categories, namely, Type I symmetric supercapacitors using p-dopable CPs, Type II asymmetric SCs with two different p-dopable CPs, and Type III symmetric SCs that can be both $\mathrm{p}$ - and n-doped. CPs can be easily deposited over the current collectors either by chemical or electrochemical methods in a binder-free manner. ${ }^{\mathbf{2 6 - 3 0}}$ Recently, conducting polymers, such as polyaniline, polypyrrole and PEDOT, have been employed in combination with carbonaceous materials and metal oxides $\left(\mathrm{MnO}_{2}, \mathrm{MoO}_{3}\right.$, and $\left.\mathrm{V}_{2} \mathrm{O}_{5}\right)$ in designing asymmetric supercapacitors. ${ }^{21,31-35}$ In this study, we investigate the electrochemical performance of an asymmetric supercapacitor made out of two conducting polymers employing a gel electrolyte.

Here, we employ a simple single-step electrodeposition method to fabricate all-conducting polymer based ASCs without employing any carbonaceous material. Specifically, we have used a conducting polymer, namely, poly(3,4-ethylenedioxythiophene) (PEDOT), due to its electrochemical activity in a wide potential window $(1.4 \mathrm{~V})$, as the negative electrode material. Simultaneously, polyaniline (PANI) was used as a positive electrode material to fabricate asymmetric solid-state supercapacitors on Au-coated plastic PEN substrates. While employing the $\mathrm{PVA} / \mathrm{H}_{2} \mathrm{SO}_{4}$ gel electrolyte, the maximum energy

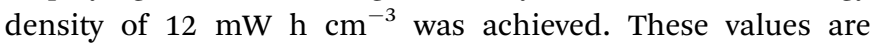
superior to asymmetric solid-sate supercapacitors based on $\mathrm{MnO}_{2}$ nanowires $/ / \mathrm{Fe}_{2} \mathrm{O}_{3}$ nanotubes electrodes $\left(E=0.55 \mathrm{~mW} \mathrm{~h} \mathrm{~cm}{ }^{-3}\right)$ and solid-state symmetric supercapacitors based on conducting polymer (ppy, $E=1 \mathrm{~mW} \mathrm{~h} \mathrm{~cm}^{-3}$; PANI/pencil/paper, $E=0.32$ $\mathrm{mW} \mathrm{h} \mathrm{cm}^{-3}$ ) electrodes. Furthermore, the tandem configuration of our asymmetric supercapacitors is used to glow a red light emitting diode.

\section{Results and discussion}

The schematic shown in Fig. 1 illustrates the fabrication of ASCs based on all-conducting polymer electrodes over the plastic PEN sheet as a supporting platform. Since PEN is insulating, a conductive coating was formed by depositing an Au metal film through thermal evaporation. Au/PEN was used as a conducting platform for the electrochemical deposition of conducting polymers such as PEDOT and PANI from their monomer solutions of EDOT and aniline with sulphuric acid as a supporting electrolyte, respectively. These PEDOT/Au/PEN and PANI/Au/

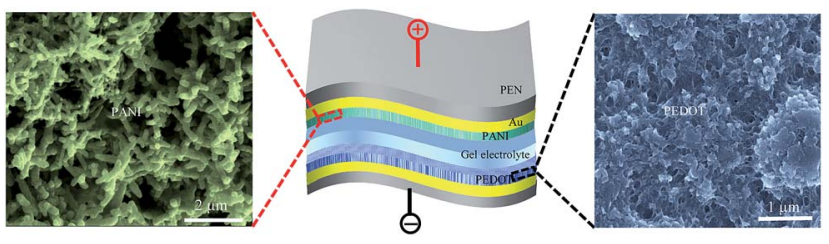

Fig. 1 Schematic illustration of the fabrication of asymmetric supercapacitors based on conducting polymer electrodes. A PANI//PEDOT ASC device with an aqueous gel electrolyte. SEM images of PANI nanofibers and nanostructured PEDOT (false colored). Thickness of each layer: PEN, $125 \mu \mathrm{m}$; Au, 150 nm; PANI, $2.5 \mu \mathrm{m}$; gel electrolyte, $60 \mu \mathrm{m}$; PEDOT, $4.5 \mu \mathrm{m}$.
PEN were employed as negative and positive electrodes, respectively (see Fig. 1), in fabricating conducting polymer based asymmetric solid state supercapacitors employing an aqueous gel electrolyte. PANI nanofibers were grown over an $\mathrm{Au}$ / PEN substrate through galvanostatic electrodeposition. Nanostructured PEDOT was grown on an Au/PEN sheet via electrodeposition under potentiostatic conditions, as shown in the SEM micrograph in Fig. 1.

The chemical composition and bonding of PEDOT and PANI grown over Au/PEN were characterized by Raman spectroscopy, and the spectra are shown in Fig. 2a and b. As shown in Fig. 2a, several characteristic peaks of PEDOT can be seen in the Raman spectrum and the assignment is as follows. The most intense peak is observed at $1440 \mathrm{~cm}^{-1}$ corresponding to the symmetric stretching of $\mathrm{C}_{\alpha}=\mathrm{C}_{\beta}$, which can also provide information about the extent of the oxidation level. ${ }^{36}$ On the other hand, the asymmetric stretching of $\mathrm{C}_{\alpha}=\mathrm{C}_{\beta}$ results in the bands at 1570 and $1509 \mathrm{~cm}^{-1}$. The peak at $1367 \mathrm{~cm}^{-1}$ corresponds to $\mathrm{C}_{\beta}-\mathrm{C}_{\beta}$ inter-ring stretching and that at $1264 \mathrm{~cm}^{-1}$ represents $\mathrm{C}_{\alpha}-\mathrm{C}_{\alpha}$ inter-ring stretching. The peak at $1105 \mathrm{~cm}^{-1}$ represents $\mathrm{C}-\mathrm{O}-\mathrm{C}$ deformation; $988 \mathrm{~cm}^{-1}$, C-C anti-symmetrical stretching mode; $702 \mathrm{~cm}^{-1}$, symmetric C-S-C deformation; $573 \mathrm{~cm}^{-1}$, oxyethylene ring deformation; and $440 \mathrm{~cm}^{-1}, \mathrm{SO}_{2}$ bending; these confirm the doping of sulfate and bisulfate anions (from sulphuric acid) in the PEDOT nanostructures during the electrochemical deposition. ${ }^{37}$ In the case of PANI/Au/PEN, the bands at 1623 and $1529 \mathrm{~cm}^{-1}$ correspond to $\mathrm{C}=\mathrm{C}$ and $\mathrm{C}=\mathrm{N}$ stretching vibrations, respectively, those at 827 and 415 correspond to $\mathrm{C}-\mathrm{H}$ deformation, while those at 1396, 1340, 1262, 1195, 741, 574 and $514 \mathrm{~cm}^{-1}$ are related to benzene ring deformations (see Fig. 2b)..$^{38}$

PEDOT exhibits lower specific capacitance when compared to other conducting polymers (PANI, Ppy) due to the larger molecular weight of the monomer unit and also the low doping level. ${ }^{39}$ However, PEDOT is electroactive with high capacitance over a wide potential window $(1.4 \mathrm{~V})$, and, in principle, can be employed as a positive as well as negative electrode material in energy storage devices (see ESI, Fig. S1 $\dagger$ ). PEDOT in its oxidized form is highly conducting and environmentally stable and, hence, p-doped PEDOT has been explored as a positive electrode material in most of the previous reports. ${ }^{2,27,29}$ Conducting polymer supercapacitors have been broadly classified into three categories, namely, Type I symmetric supercapacitors using

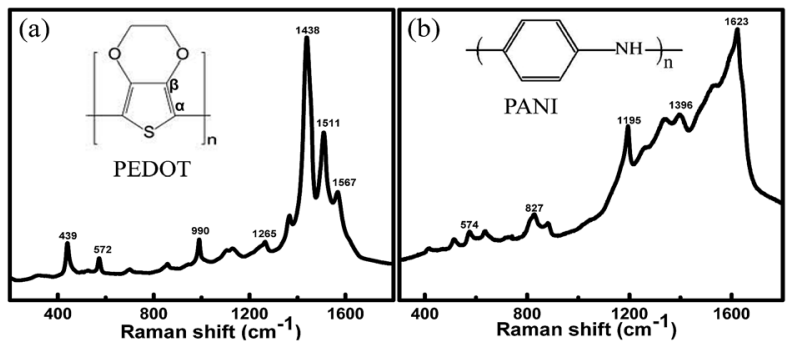

Fig. 2 (a) and (b) Raman spectra of electrochemically deposited PEDOT and PANI from their corresponding monomer aqueous solutions, respectively. 
p-dopable conducting polymers (CPs), Type II asymmetric supercapacitors with two different p-dopable CPs, and Type III symmetric supercapacitors that can be both p- and n-doped. ${ }^{1}$ Further, n-doped (de-doped) CPs are less conducting and more unstable, limiting the usage of CPs as an anode material. ${ }^{1,2}$ PEDOT has been a promising electrode material for Type I and asymmetric Type II supercapacitors because of its high stability in the oxidation state. ${ }^{40,41}$ However, it has been demonstrated that the various doped states of PEDOT can be used as cathode and anode for making rechargeable batteries. ${ }^{42,43}$ Recently, it was demonstrated that improving the conductivity of PEDOT:PSS by solvent annealing can induce electrochemical activity in a wider potential window by shifting the reduction potential towards more negative values. ${ }^{44} \mathrm{~J}$. Ahonen et al. have performed $\mathrm{p}$ - and $\mathrm{n}$-doping of PEDOT in an anhydrous organic medium and observed that PEDOT gets n-doped beyond a potential of $-1 \mathrm{~V} \cdot{ }^{45}$ Further, they found that the reduced form of PEDOT is not stable even in an extremely dry oxygen-free environment, which may severely hamper the use of PEDOT in the extreme negative potential windows. ${ }^{45}$ However, in our case, we have observed that PEDOT is quite stable until the potential of $-0.5 \mathrm{~V}$. Beyond the potential of $-0.5 \mathrm{~V}$, we have observed a sudden rise in the negative current, which can be attributed to the hydrogen evolution reaction as the electrolyte medium is 1 $\mathrm{M} \mathrm{H}_{2} \mathrm{SO}_{4}$ (see Fig. S1a, ESI†). Hence, the conductivity, higher degree of polymerization and electron donating nature of alkoxy moieties of PEDOT make it usable as an anode material by shifting the reduction potential towards higher negative potentials, without getting it into the n-doped state. ${ }^{44,45}$

We have investigated the electrochemical behavior of $\mathrm{PEDOT} / \mathrm{Au} / \mathrm{PEN}$ electrode in the negative potential window $(0.3$ to $-0.5 \mathrm{~V}$ ) in $1 \mathrm{M} \mathrm{H}_{2} \mathrm{SO}_{4}$ electrolyte in a 3-electrode configuration. As shown in Fig. 3a, the CV curves are rectangular in shape with the appearance of broad redox peaks due to the electrochemical doping of PEDOT by the electrolyte ions. However, it is difficult to assign the oxidation/reduction (doping/dedoping)
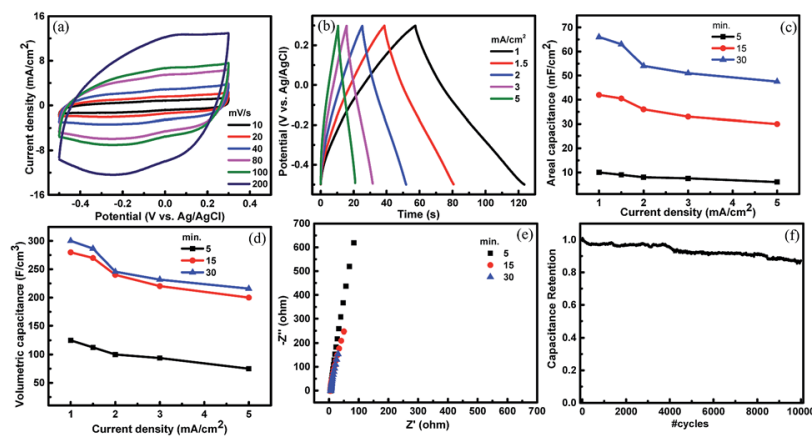

Fig. 3 Electrochemical behavior of PEDOT/Au/PEN electrode in a 3electrode configuration in the negative potential window. (a) Cyclic voltammograms (CVs) and (b) galvanostatic charge-discharge (CD) curves for PEDOT (30 min)/Au/PEN electrode in the negative potential window. (c) and (d) Areal and volumetric capacitance of PEDOT deposited for different time vs. current density values. (e) Nyquist plots of various PEDOT/Au/PEN electrodes. (d) Electrochemical stability of the PEDOT/Au/PEN electrode over 10000 cycles at a current density of $2 \mathrm{~mA} \mathrm{~cm}^{-2}$. due to the short-life times of the intermediate unstable species. Chen et al. have performed fast scan cyclic voltammetry at low temperatures to understand the electrochemical behavior of PEDOT. ${ }^{46}$ PEDOT is quite stable until the potential of $-0.5 \mathrm{~V}$ due to its doped state, which may get de-doped below $-0.5 \mathrm{~V}$, resulting in the loss of capacitance due to diminished conductivity. ${ }^{47,48}$ The charge-discharge curves are seen as linear in the potential window of 0.3 to $-0.5 \mathrm{~V}$ at different current densities from 1 to $5 \mathrm{~mA} \mathrm{~cm}{ }^{-2}$ (see Fig. 3b). The mass loading of the PEDOT deposits was found to be $0.08,0.251$ and $0.37 \mathrm{mg} \mathrm{cm}^{-2}$ after 5,15 and 30 minutes of deposition, respectively. The areal capacitance was calculated from the slope of the discharge curve at each current density. As shown in Fig. 3c, the areal capacitance was found to be $66 \mathrm{mF} \mathrm{cm}{ }^{-2}$ at a current density of $1 \mathrm{~mA} \mathrm{~cm}^{-2}$ for the 30 minute deposited PEDOT sample. This value was gradually decreased to $47 \mathrm{mF} \mathrm{cm}{ }^{-2}$ at a current density of $5 \mathrm{~mA} \mathrm{~cm}{ }^{-2}$. In the case of the 5 minute deposited PEDOT sample, the areal capacitance was varying from 10 to 6 $\mathrm{mF} \mathrm{cm}{ }^{-2}$ at different current density values (see Fig. $3 \mathrm{c}$ and S2, ESI $\dagger$ ). Similarly, the areal capacitance for the 15 minute deposited PEDOT sample was found to vary from 42 to $30 \mathrm{mF} \mathrm{cm}$. The typical values of gravimetric capacitance were found to be 125, 168 and $175 \mathrm{~F} \mathrm{~g}^{-1}$ for the 5, 15 and 30 minutes PEDOT samples (at a current density of $1 \mathrm{~mA} \mathrm{~cm}^{-2}$ ), respectively. The volumetric capacitance was calculated by dividing the areal capacitance with the corresponding thickness of PEDOT deposited for different times. The variation in the volumetric capacitance at different current densities for the 5, 15 and 30 minute deposited PEDOT samples are shown in Fig. 3d. It was observed that the volumetric capacitance was increasing from 125 to $280 \mathrm{~F} \mathrm{~cm}^{-3}$ at a current density of $1 \mathrm{~mA} \mathrm{~cm}^{-2}$ as the deposition time increased from 5 to 15 minutes. In the case of the 30 minute deposited PEDOT sample, the volumetric capacitance was found to be $300 \mathrm{~F} \mathrm{~cm}^{-3}$, indicating that the extended deposition beyond a critical thickness (in this case, $1.5 \mu \mathrm{m}$ ) may not lead to a corresponding increase in the volumetric capacitance. Although there is an enhancement in the areal capacitance of 1.5 times for the 30 minute deposited PEDOT sample when compared to the 15 minute deposited PEDOT sample, not much improvement was found in the volumetric and gravimetric capacitances of the two samples. This volumetric capacitance is superior when compared to activated carbon and some of the carbonaceous materials that have been employed as negative electrodes in making asymmetric supercapacitors. ${ }^{15}$ The Nyquist plots of PEDOT deposited for different times (5-30 min) are shown in Fig. 3e. The absence of a semicircle in the high frequency regions indicates negligible charge-transfer resistance across the PEDOT/electrolyte interface. The electrochemical stability of the PEDOT electrode was tested by charging and discharging for 10000 cycles in the potential window of 0.3 to $-0.5 \mathrm{~V}$. The capacitance is found to be retained up to $87 \%$ after 10000 cycles, as shown in Fig. 3f. A solid state PEDOT symmetric device was made by assembling two PEDOT/Au/PEN electrodes using the $\mathrm{PVA} / \mathrm{H}_{2} \mathrm{SO}_{4}$ gel electrolyte (see ESI, Fig. S2 $\dagger$ ). A symmetric solid state PEDOT/Au/ $\mathrm{PEN} / / \mathrm{PEDOT} / \mathrm{Au} / \mathrm{PEN}$ device exhibits a volumetric stack capacitance of $20 \mathrm{~F} \mathrm{~cm}^{-3}$ at a current density of $0.25 \mathrm{~mA} \mathrm{~cm}^{-2}$, which 
is in close agreement with that of previously reported PEDOT solid state devices on a carbon fiber paper. ${ }^{49}$

To investigate the electrochemical performance of PANI in the positive potential window ( 0 to $0.8 \mathrm{~V}$ ), PANI was electrodeposited on $\mathrm{Au} / \mathrm{PEN}$ followed by measuring its capacitive behavior in a 3-electrode configuration in $1 \mathrm{M} \mathrm{H}_{2} \mathrm{SO}_{4}$ electrolyte. As marked in Fig. 4a, CV scans at different scan rates (10-200 $\mathrm{mV} \mathrm{s}^{-1}$ ) exhibit two pairs of redox peaks, $\mathrm{A}_{1} / \mathrm{C}_{1}$ and $\mathrm{A}_{2} / \mathrm{C}_{2}$ (see ESI, Fig. S4 $\dagger)$. The redox pair $\left(A_{1} / C_{1}\right)$ at a lower potential can be attributed to the redox transition of PANI between its leucoemeraldine form (semiconducting) to a polaronicemeraldine form (conducting), while the redox peaks at a higher potential can be due to the reversible formation of redox pairs such as $p$ benzo/hydroquinone and $p$-aminophenol/benzoquinoneimine. ${ }^{50,51}$ Due to pseudocapacitive nature of PANI, it exhibits much higher values of capacitance when compared with other conducting polymers. The deviation from the triangular shape of the charge-discharge curves is due to the Faradaic contribution of PANI in the electrolyte medium (aqueous $\mathrm{H}_{2} \mathrm{SO}_{4}$ ), indicating the pseudocapacitive nature of the PANI/Au/PEN electrode, as shown in Fig. $4 \mathrm{~b}$. The typical mass loadings of PANI were found to be $0.15,0.3$ and $0.95 \mathrm{mg}$ after 3,5 and 10 minutes of deposition, respectively. As shown in Fig. 4c, the areal capacitance was increasing gradually from 40 to $110 \mathrm{mF}$ $\mathrm{cm}^{-2}$ at a current density of $1 \mathrm{~mA} \mathrm{~cm}{ }^{-2}$ when the deposition time increased from 3 to 5 minutes. This corresponds to the specific mass capacitance of 275 and $362 \mathrm{~F} \mathrm{~g}^{-1}$ for the 3 and 5 minutes deposited PANI samples, respectively. The maximum areal capacitance was found to be $450 \mathrm{mF} \mathrm{cm}^{-2}$ (mass specific capacitance of $474 \mathrm{~F} \mathrm{~g}^{-1}$ ) for the 10 minute deposited PANI sample at a current density of $1 \mathrm{~mA} \mathrm{~cm}{ }^{-2}$. These results are in agreement with the literature reports. ${ }^{24,25}$ The volumetric capacitance of $366 \mathrm{~F} \mathrm{~cm}^{-3}$ (corresponding areal capacitance of $110 \mathrm{mF} \mathrm{cm}^{-2}$ ) was obtained for the 5 minute deposited PANI sample.

Further, two symmetrical PANI/Au/PEN electrodes were glued together with the aid of the $\mathrm{PVA} / \mathrm{H}_{2} \mathrm{SO}_{4}$ gel electrolyte in order to fabricate a solid state symmetric PANI supercapacitor.
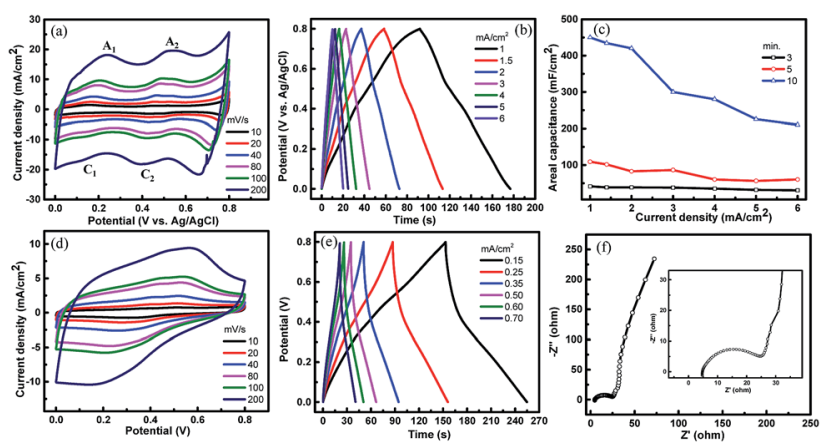

Fig. 4 (a) $C V$ and (b) $C D$ curves of PANI/Au/PEN electrode in $1 \mathrm{M}$ $\mathrm{H}_{2} \mathrm{SO}_{4}$ in a 3-electrode configuration. (c) Variation in the areal capacitance with current density for the PANI samples deposited for different times. (d) CV and (e) CD curves of PANI/Au/PEN//PANI/Au/ PEN solid state symmetric supercapacitor using $\mathrm{PVA} / \mathrm{H}_{2} \mathrm{SO}_{4}$ gel electrolyte. (f) Nyquist plot for the PANI solid state device. Inset shows the high frequency portion of the spectrum.
Cyclic voltammetry and charge-discharge curves for the PANI solid state devices are shown in Fig. $4 \mathrm{~d}$ and e. The CV scans of the solid state device at different scan rates $\left(10-200 \mathrm{mV} \mathrm{s}^{-1}\right)$ exhibit broad redox peaks due to the quasi solid state nature of the gel electrolyte, unlike the liquid $\mathrm{H}_{2} \mathrm{SO}_{4}$ electrolyte where the distinct redox peaks have been marked (see Fig. 4a). Similarly, the charge-discharge curves were found to be nonlinear and the areal cell stack capacitance was found to be $29 \mathrm{mF} \mathrm{cm}^{-2}$ at a current density of $0.15 \mathrm{~mA} \mathrm{~cm}{ }^{-2}$ for the PANI symmetric solid state device (see Fig. 4e). This corresponds to a volumetric stack capacitance of $57 \mathrm{~F} \mathrm{~cm}^{-3}$ for the 5 minute deposited PANI solid state symmetric supercapacitor. The Nyquist plot for the solid state device is shown in Fig. 4f. The vertical nature of the spectrum in the low frequency region indicates the capacitive behavior of the solid state device. In the high frequency region, the spectrum is seen with a semicircle due to the charge transfer resistance offered at the electrode/electrolyte interface with a solution resistance of $5 \Omega$ (in this case, a gel electrolyte).

The representative CVs of PEDOT (30 minute) and PANI (5 minute) in the negative and positive potential windows at a scan rate of $80 \mathrm{mV} \mathrm{s}^{-1}$ are shown in Fig. 5a. The charge on both the electrodes was matched by balancing the volumetric capacitance of the PEDOT and PANI electrodes. The geometric volume of the electrodes was balanced by the following equation: $V_{+} / V_{-}=\left(C_{-} \times \Delta E_{-}\right) /\left(C_{+} \times \Delta E_{+}\right)$, where $V_{-}, V_{+}, C_{-}$and $C_{+}$, and $\Delta E_{-}$and $\Delta E_{+}$are the volume and the capacitance and potential windows of the negative and positive electrodes, respectively. Since the overlapping physical area of the positive and negative electrodes is the same, the variable parameter is the thickness of the electrodes. The thickness ratio, $t_{+} / t_{-}$, was found to be 0.51 . Obviously, the volume of the electrodes (mostly, thickness) was controlled through the electrodeposition time of PEDOT and PANI electrodes. An asymmetric solid
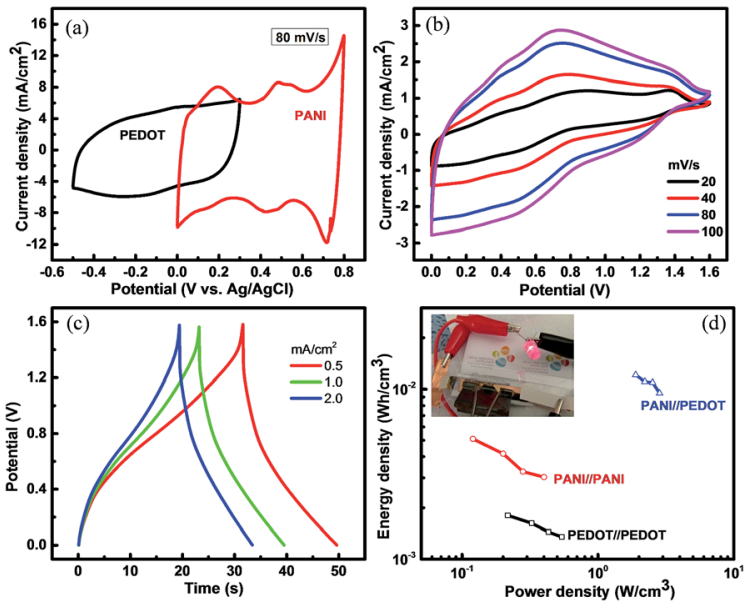

Fig. 5 (a) CV curves of PEDOT/Au/PEN and PANI/Au/PEN electrodes in different potential windows, measured in $1 \mathrm{M} \mathrm{H}_{2} \mathrm{SO}_{4}$ in a 3-electrode configuration at a scan rate of $80 \mathrm{mV} \mathrm{s}^{-1}$. (b) CVs of the asymmetric solid state device at different scan rates. (c) Charge-discharge curves of the ASC at different current densities. (d) Ragone plot for the symmetric and asymmetric solid state conducting polymer supercapacitors made in this study. Inset shows the glowing of an LED using three ASC cells connected in series. 
state supercapacitor was fabricated by employing the optimized thickness of PEDOT and PANI electrodes (see the performance of unoptimised ASC, Fig. S5, ESI $\dagger$ ). The total thickness of PEDOT (thickness: $4.5 \mu \mathrm{m}$ ) and PANI (thickness: $2.5 \mu \mathrm{m}$ ) was found to be $7 \mu \mathrm{m}$ in fabricating the ASC device. The electrodes were wetted with a thin layer of $\mathrm{PVA} / \mathrm{H}_{2} \mathrm{SO}_{4}$ gel electrolyte followed by assembling the electrodes to form the ASC device. The CVs shown in Fig. 5b were recorded at different scan rates in the potential window of $0-1.6 \mathrm{~V}$ (see Fig. S6, ESI $\dagger$ ). The ASC device exhibits redox behavior due to the redox activity of the conducting polymer electrodes in the electrolyte media. The charge-discharge curves of the ASC device were recorded in the potential window of 0 to $1.6 \mathrm{~V}$ at different current densities $\left(0.5-2 \mathrm{~mA} \mathrm{~cm}^{-2}\right)$, as shown in Fig. 5c. The curvature in the CD profiles is due to the Faradaic contribution from both the conducting polymer electrodes. This ASC device exhibited volumetric stack capacitance of $34 \mathrm{~F}$ $\mathrm{cm}^{-3}$ at a current density of $0.5 \mathrm{~mA} \mathrm{~cm}{ }^{-2}$. The cycling stability of the ASC was tested over 10000 cycles at a current density of $2 \mathrm{~mA} \mathrm{~cm}^{-2}$, which showed capacitance retention up to $80 \%$ (see ESI, Fig. S6d $\dagger$ ). ${ }^{52}$ The energy and power densities of various solid state supercapacitors fabricated in this study are plotted and shown in the Ragone plot in Fig. 5d. The ASC solid state PANI//PEDOT device exhibits the highest energy

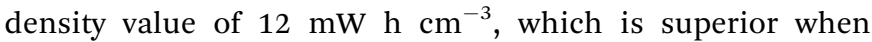
compared to the symmetric PEDOT $\left(E=2 \mathrm{~mW} \mathrm{~h} \mathrm{~cm}^{-3}\right)$ and PANI $\left(E=5 \mathrm{~mW} \mathrm{~h} \mathrm{~cm}^{-3}\right)$ based solid state supercapacitors.

The energy density of the solid state ASC is higher by an order of magnitude to that of graphene oxide and onion like carbon supercapacitors $\left(0.8-1 \mathrm{~mW} \mathrm{~h} \mathrm{~cm}^{-3}\right)$ and comparable to that of aqueous activated carbon based supercapacitors $(10 \mathrm{~mW}$ $\mathrm{h} \mathrm{cm}^{-3}$ ) with a power density of $1300 \mathrm{~mW} \mathrm{~cm}^{-3}$. 3,54 This solid state ASC device exhibits the maximum power density of $2.8 \mathrm{~W} \mathrm{~cm}^{-3}$ at an energy density of $9 \mathrm{~mW} \mathrm{~h} \mathrm{~cm}^{-3}$. Further, this ASC PANI// PEDOT exhibits much higher energy density over some of the symmetric supercapacitors based on conducting polymer electrodes such as ppy/paper $\left(E=1 \mathrm{~mW} \mathrm{~h} \mathrm{~cm}^{-3}, P=270 \mathrm{~mW} \mathrm{~cm}^{-3}\right)^{28}$ and also ASC solid state devices based on $\mathrm{MnO}_{2}$ nanowires// $\mathrm{Fe}_{2} \mathrm{O}_{3}$ nanotubes $\left(E=0.55 \mathrm{~mW} \quad \mathrm{~h} \quad \mathrm{~cm}^{-3}\right),{ }^{22} \quad \mathrm{VO}_{x} / / \mathrm{VN}$ $\left(E=0.61 \mathrm{~mW} \mathrm{~h} \mathrm{~cm}{ }^{-3}\right)^{55}$ and $\mathrm{H}-\mathrm{TiO}_{2} @ \mathrm{MnO}_{2} \mathrm{NWs} / / \mathrm{H}-\mathrm{TiO}_{2} @ \mathrm{C}$ core shell NWs $\left(E=0.3 \mathrm{~mW} \mathrm{~h} \mathrm{~cm}{ }^{-3}\right) .{ }^{56}$ As the typical mass loading of conducting polymer electrodes was found to be less than $1 \mathrm{mg} \mathrm{cm}{ }^{-2}$, we have estimated the volumetric energy and power densities of the solid state devices. ${ }^{57}$ The higher values of energy density for the conducting polymer ASCs can be attributed to the redox contribution from both the electrodes, which was evident from the electrochemical behavior of the device. The electrochemical activity of the electrode materials in a given electrolyte medium and the extent of wettability decides the performance of the ASC (see ESI, Table S1 $\dagger$ ). Further, three ASC cells were connected in series to demonstrate them as a power source by glowing a red light emitting diode (see inset of Fig. 5d).

It is worth mentioning that Crispin and co-workers have used PEDOT as an anode and cathode for fabricating an allpolymer based battery with different doping levels. ${ }^{\mathbf{2 2 , 4 3}}$ Employing the same conducting polymer with different doping states comes under the category of Type III-based devices. As both the electrodes are in the charged state, the open circuit potential can be very high, which may deliver high specific energy and power densities. These electrodes have been tested in organic electrolytes rather than aqueous electrolytes. In our case, we relied on two different CPs with complimentary potential windows for the asymmetric design (Type II devices). Since PANI shows high electrochemical activity in an acidic medium (requires protons for redox reactions), we have employed an aqueous acid electrolyte for fabricating these conducting polymer based ASC devices.

Recent reports have demonstrated the usage of PANI-porous nanofiber electrodes and PEDOT in designing symmetric and asymmetric supercapacitors by adopting composite materials. ${ }^{58-60}$ In contrast to the above studies, we have employed two different CPs as electrode materials in an asymmetric design in order to utilize their complimentary potential windows in extending the working potential window of aqueous based electrolytes beyond $1.23 \mathrm{~V}$, which further increases the energy and power densities of these ASC devices $\left(E=1 / 2 C V^{2}\right.$ and $P=V^{2} / 4 R$ ). As the symmetric device based on PEDOT (both anode and cathode) utilizes different doping states of the same $\mathrm{CP}$, delivering higher open circuit potential helps in powering an LED bulb for almost up to 3 minutes. ${ }^{42}$ But in our case, the open circuit potentials are small as we use two different CPs in the doped state and we relied on charging by applying potential externally, which gets harnessed during the discharge process in powering an LED. In this case, the charge and discharge kinetics are relatively faster compared to the all-polythiophene rechargeable batteries reported recently. ${ }^{42}$ The advantages of our new devices include higher potential window (up to $1.6 \mathrm{~V}$ ) of aqueous media and faster charge-discharge rates with good cycling stability.

\section{Conclusions}

Solid state asymmetric supercapacitors were fabricated by employing the conducting polymers PEDOT and PANI as a negative and positive electrode, respectively, and $\mathrm{PVA} / \mathrm{H}_{2} \mathrm{SO}_{4}$ gel electrolyte. The asymmetric devices showed the maximum power density of $2.8 \mathrm{~W} \mathrm{~cm}^{-3}$ at an energy density of $9 \mathrm{~mW} \mathrm{~h} \mathrm{~cm}{ }^{-3}$, which is much higher than other asymmetric solid-state devices based on $\mathrm{MnO}_{2}$ nanowires $/ / \mathrm{Fe}_{2} \mathrm{O}_{3}$ nanotubes $\left(E=0.55 \mathrm{~mW} \mathrm{~h} \mathrm{~cm}^{-3}\right)$ and solid-state devices based on ppy/paper $\left(E=1 \mathrm{~mW} \mathrm{~h} \mathrm{~cm}^{-3}\right)$

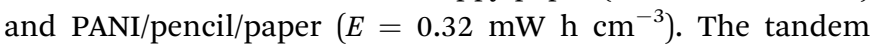
configuration of these asymmetric devices was utilised to demonstrate the glowing of a light emitting diode for about 1 minute after charging for 10 seconds.

\section{Experimental section}

\section{Materials}

Chemicals were used as received without further purification. Analytical grade $\mathrm{H}_{2} \mathrm{SO}_{4}$ and 3,4-ethylenedioxythiophene (EDOT), aniline (ANI) and surface active reagent sodium dodecyl sulphate (SDS, $\mathrm{CH}_{3}\left(\mathrm{CH}_{2}\right)_{11} \mathrm{OSO}_{3} \mathrm{Na}$ ) and polyvinyl alcohol (PVA) were purchased from Sigma-Aldrich. 


\section{Electrode preparation and electrochemical deposition}

Polyethylene naphthalate (PEN) plastic sheets (thickness: 125 $\mu \mathrm{m})$ were employed as substrates for making the conducting polymer based supercapacitors. These PEN sheets were coated with $150 \mathrm{~nm} \mathrm{Au/10} \mathrm{nm} \mathrm{Ti} \mathrm{as} \mathrm{conducting} \mathrm{layers} \mathrm{for} \mathrm{electro-}$ depositing the conducting polymers. The electrodeposition was performed in a standard 3-electrode configuration in a glass cell consisting of $\mathrm{Au} / \mathrm{PEN}$ as the working electrode and a platinum wire counter electrode and $\mathrm{Ag} / \mathrm{AgCl}$ as the reference electrode at room temperature. An electrolyte with $10 \mathrm{mM}$ EDOT + $1 \mathrm{M}$ $\mathrm{H}_{2} \mathrm{SO}_{4}+10 \mathrm{mM}$ SDS was employed to deposit PEDOT using $\mathrm{CHI}$ $660 \mathrm{D}$ Electrochemical Workstation. The role of the anionic surfactant is to improve the miscibility of the EDOT monomers in water, which also lowers the oxidation potential below $1 \mathrm{~V}^{30} \mathrm{~A}$ constant anodic potential of $0.9 \mathrm{~V}(v s . \mathrm{Ag} / \mathrm{AgCl})$ was used for different times of 5, 15 and 30 minutes to deposit PEDOT of different thicknesses over Au/PEN. After the electrochemical deposition, the samples were thoroughly washed with DI water in order to remove the surfactant molecules adsorbed on the surface. Polyaniline (PANI) was electrodeposited using $0.1 \mathrm{M}$ aniline $+1 \mathrm{M} \mathrm{H}_{2} \mathrm{SO}_{4}$ as the electrolyte by galvanostatic electrodeposition at a constant current density of $0.5 \mathrm{~mA} \mathrm{~cm}{ }^{-2}$ for different times. ${ }^{26}$

\section{Material characterization}

The surface morphology and microstructure were imaged by a scanning electron microscope (SEM) (Nova Nano 630 instrument, FEI Co., The Netherlands). The energy-dispersive spectroscopy (EDS) analysis was performed with an EDAX Genesis instrument (Mahwah, NJ) attached to the SEM column. Au coating was done by thermal evaporation of $150 \mathrm{~nm}$ thick Au on the PEN substrate. The film thicknesses were measured using a Veeco Dektak 150 surface profilometer. Raman spectroscopy measurements were carried out on the PEDOT samples using a micro-Raman spectrometer (LabRAM ARAMIS, Horiba-Jobin Yvon). Raman spectra were acquired with notch filters cutting at $100 \mathrm{~cm}^{-1}$ using a cobalt laser $(473 \mathrm{~nm}, 5 \mathrm{~mW}$ at source $)$ and a laser spot size of $1.5 \mu \mathrm{m}$.

\section{Preparation of aqueous gel electrolytes}

The electrochemical properties of PEDOT/Au/PEN and PANI/Au/ PEN were investigated in both 3- and 2-electrode configurations in $1 \mathrm{M} \mathrm{H}_{2} \mathrm{SO}_{4}$ and PVA/ $\mathrm{H}_{2} \mathrm{SO}_{4}$ aqueous gel electrolytes. The PVA/ $\mathrm{H}_{2} \mathrm{SO}_{4}$ gel electrolyte was prepared as follows: $1 \mathrm{~g}$ of $\mathrm{H}_{2} \mathrm{SO}_{4}$ was added into $10 \mathrm{~mL}$ of deionized water, followed by adding $1 \mathrm{~g}$ of PVA powder. The whole mixture was heated to $85{ }^{\circ} \mathrm{C}$ while stirring until the solution became clear.

\section{Fabrication and characterization of asymmetric supercapacitor devices}

Solid-state symmetric and asymmetric supercapacitor devices were fabricated by applying the polymer gel electrolyte on the individual negative PEDOT and positive PANI electrodes followed by assembling together and allowing it to dry out the excess water. Cyclic voltammetry (CV), galvanostatic charge- discharge (CD), and electrochemical impedance spectroscopy (EIS) were performed on an electrochemical workstation (model 660D, CH Instruments, Austin, TX, USA). The CVs were tested in different potential windows at varied scan rates, ranging from 10 to $200 \mathrm{mV} \mathrm{s}^{-1}$. The CDs were measured at different current densities from 0.1 to $5 \mathrm{~mA} \mathrm{~cm}{ }^{-2}$. The EIS was performed in the frequency range from $100 \mathrm{kHz}$ to $0.1 \mathrm{~Hz}$ at the open circuit potential by applying a small sinusoidal potential of $10 \mathrm{mV}$ signal. All the measurements were carried out at room temperature.

\section{Acknowledgements}

Research reported in this publication was supported by King Abdullah University of Science and Technology (KAUST). Authors thank the Advanced Nanofabrication, Imaging and Characterization Laboratory at KAUST for their excellent support. Ruiqi Wang wants to thank SRSI for giving this opportunity and Ms. Tsvetanka B. Sendova for her assistance. NK acknowledges the support from SABIC Postdoctoral Fellowship.

\section{References}

1 M. E. Abdelhamid, A. P. O'Mullaneb and G. A. Snook, RSC Adv., 2015, 5, 11611-11626.

2 L. Nyholm, G. Nyström, A. Mihranyan and M. Strømme, Adv. Mater., 2011, 23, 3751-3769.

3 C. Meng, C. Liu, L. Chen, C. Hu and S. Fan, Nano Lett., 2010, 10, 4025-4031.

4 P. Simon and Y. Gogotsi, Nat. Mater., 2008, 7, 845.

5 M. Winter and R. J. Brodd, Chem. Rev., 2004, 104, 4245-4270.

6 B. E. Conway, Electrochemical Supercapacitors, Scientific, Fundamentals and Technological Applications, Plenum, New York, 1999.

7 G. Wang, L. Zhang and J. Zhang, Chem. Soc. Rev., 2012, 41, 797-828.

8 Y. Zhai, Y. Dou, D. Zhao, P. F. Fulvio, R. T. Mayes and S. Dai, Adv. Mater., 2011, 23, 4828-4850.

9 Y. Huang, J. Liang and Y. Chen, Small, 2012, 8, 1805-1834.

10 H. Nishihara and T. Kyotani, Adv. Mater., 2012, 24, 44734498.

11 S. L. Candelaria, Y. Shao, W. Zhou, X. Li, J. Xiao, J.-G. Zhang, Y. Wang, J. Liu, J. Li and G. Cao, Nano Energy, 2012, 1, 195220.

12 J. Jiang, Y. Li, J. Liu, X. Huang, C. Yuan and X. W. Lou, Adv. Mater., 2012, 24, 5166-5180.

13 M. Zhi, C. Xiang, J. Li, M. Li and N. Wu, Nanoscale, 2013, 5, 72-88.

14 R. Ramya, R. Sivasubramanian and M. V. Sangaranarayanan, Electrochim. Acta, 2013, 101, 109-129.

15 F. Wang, S. Xiao, Y. Hou, C. Hu, L. Liu and Y. Wu, RSC Adv., 2013, 3, 13059-13084.

16 H. C. Gao, F. Xiao, C. B. Ching and H. W. Duan, ACS Appl. Mater. Interfaces, 2012, 4, 7020-7026.

17 D. H. Nagaraju, Q. Wang, P. Beaujuge and H. N. Alshareef, J. Mater. Chem. A, 2014, 2, 17146-17152. 
18 Z. Tang, C. H. Tang and H. Gong, Adv. Funct. Mater., 2012, 22, 1272-1278.

19 Z. S. Wu, W. C. Ren, D. W. Wang, F. Li, B. L. Liu and H. M. Cheng, ACS Nano, 2010, 4, 5835-5842.

20 C. Yang, J. Shen, C. Wang, H. Fei, H. Bao and G. Wang, J. Mater. Chem. A, 2014, 2, 1458-1464.

21 Y. Hou, L. Chen, P. Liu, J. Kang, T. Fujita and M. Chen, J. Mater. Chem. A, 2014, 2, 10910-10916.

22 P. Yang, Y. Ding, Z. Lin, Z. Chen, Y. Li, P. Qiang, M. Ebrahimi, W. Mai, C. P. Wong and Z. L. Wang, Nano Lett., 2014, 14, 731-736.

23 Q. T. Qu, Y. S. Zhu, X. W. Gao and Y. Wu, Adv. Energy Mater., 2012, 2, 950-955.

24 X. Lu, Y. Zeng, M. Yu, T. Zhai, C. Liang, S. Xie, M.-S. Balogun and Y. Tong, Adv. Mater., 2014, 26, 3148-3155.

25 T. Zhai, X. Lu, Y. Ling, M. Yu, G. Wang, T. Liu, C. Liang, Y. Tong and Y. Li, Adv. Mater., 2014, 26, 5869-5875.

26 K. Wang, H. Wu, Y. Meng and Z. Wei, Small, 2014, 10, 14-31.

27 L. Yuan, X. Xiao, T. Ding, J. Zhong, X. Zhang, Y. Shen, B. Hu, Y. Huang, J. Zhou and Z. L. Wang, Angew. Chem., Int. Ed., 2012, 51, 4934-4938.

28 L. Yuan, B. Yao, B. Hu, K. Huo, W. Chen and J. Zhou, Energy Environ. Sci., 2013, 6, 470-476.

29 N. Kurra, J. Park and H. N. Alshareef, J. Mater. Chem. A, 2014, 2, 17058-17065.

30 S. Patra and N. Munichandraiah, J. Appl. Polym. Sci., 2007, 106, 1160-1171.

31 J. Shen, C. Yang, X. Li and G. Wang, ACS Appl. Mater. Interfaces, 2013, 5, 8467-8476.

32 X. Xiao, T. Ding, L. Yuan, Y. Shen, Q. Zhong, X. Zhang, Y. Cao, B. Hu, T. Zhai, L. Gong, et al., Adv. Energy Mater., 2012, 2, 1328-1332.

33 H. Peng, G. Ma, J. Mu, K. Sun and Z. Lei, J. Mater. Chem. A, 2014, 2, 10384.

34 J. Liu, L. Zhang, H. B. Wu, J. Lin, Z. Shen and X. W. (David) Lou, Energy Environ. Sci., 2014, 7, 3709.

35 Y. Zhou, H. Xu, N. Lachman, M. Ghaffari, S. Wu, Y. Liu, A. Ugur, K. K. Gleason, B. L. Wardle and Q. M. Zhang, Nano Energy, 2014, 9, 176-185.

36 P. Subramanian, N. Clark, B. Winther-Jensen, D. MacFarlane and L. Spiccia, Aust. J. Chem., 2009, 62, 133-139.

37 B. Winther-Jensen and K. West, Macromolecules, 2004, 37, 4538-4543.

38 G. Cai, J. Tu, D. Zhou, J. Zhang, Q. Xiong, X. Zhao, X. Wang and C. Gu, J. Phys. Chem. C, 2013, 117, 15967-15975.

39 G. A. Snook and G. Z. Chen, J. Electroanal. Chem., 2008, 612, 140-146.
40 J. A. Irvin and J. D. Stenger-Smith, in Handbook of Conducting Polymers: Processing and Applications, ed. T. A. Skotheim and J. R. Reynolds, CRC Press, Boca Raton, 3rd edn, 2007, 9/1.

41 M. Anna, D. Österholm, E. Shen, A. L. Dyer and J. R. Reynolds, ACS Appl. Mater. Interfaces, 2013, 5, 1343213440.

42 D. Aradilla, F. Estrany, F. Casellas, J. I. Iribarren and C. Alemán, Org. Electron., 2014, 15, 40-46.

43 Y. Xuan, M. Sandberg, M. Berggren and X. Crispin, Org. Electron., 2012, 13, 632-637.

44 H.-S. Park, S.-J. Ko, J.-S. Park, J. Y. Kim and H.-K. Song, Sci. Rep., 2013, 3, 976-983.

45 H. J. Ahonen, J. Lukkari and J. Kankare, Macromolecules, 2000, 33, 6787-6793.

46 X. Chen and O. Inganas, J. Phys. Chem., 1996, 100, 1520215206.

47 J. Duay, E. Gillette, R. Liu and S. B. Lee, Phys. Chem. Chem. Phys., 2012, 14, 3329-3337.

48 L. Bert, et al., Adv. Mater., 2003, 15, 855.

49 B. Anothumakkool, A. Torris, S. N. Bhange, M. V. Badiger and S. Kurungot, Nanoscale, 2014, 6, 5944-5952.

50 D. W. Wang, F. Li, J. P. Zhao, W. C. Ren, Z. G. Chen, J. Tan, Z. S. Wu, I. Gentle, G. Q. Lu and H. M. Cheng, ACS Nano, 2009, 3, 1745-1752.

51 D. E. Stilwell and S. M. Park, J. Electrochem. Soc., 1988, 135, 2254-2262.

52 G. A. Snook, G. J. Wilson and A. G. Pandolfo, J. Power Sources, 2009, 186, 216-223.

53 D. Pech, M. Brunet, H. Durou, P. Huang, V. Mochalin, Y. Gogotsi, P. L. Taberna and P. Simon, Nat. Nanotechnol., 2010, 5, 651-654.

54 W. Gao, N. Singh, L. Song, Z. Liu, A. L. M. Reddy, L. Ci, R. Vajtai, Q. Zhang, B. Wei and P. M. Ajayan, Nat. Nanotechnol., 2011, 6, 496-500.

55 X. Lu, M. Yu, T. Zhai, G. Wang, S. Xie, T. Liu, C. Liang, Y. Tong and Y. Li, Nano Lett., 2013, 13, 2628-2633.

56 X. Lu, M. Yu, G. Wang, T. Zhai, S. Xie, Y. Ling, Y. Tong and Y. Li, Adv. Mater., 2013, 25, 267.

57 M. D. Stoller and R. S. Ruoff, Energy Environ. Sci., 2010, 3, 1294-1301.

58 M. Dirican, M. Yanilmaza and X. Zhang, RSC Adv., 2014, 4, 59427-59435.

59 D. Aradilla, D. Azambuja, F. Estrany, M. T. Casas, C. A. Ferreirae and C. Alemán, J. Mater. Chem., 2012, 22, 13110-13122.

60 L. Fan, N. Zhang and K. Sun, Chem. Commun., 2014, 50, 6789-6792. 